\title{
Brownian and advective dynamics in microflow studied by coherent $X$-ray scattering experiments
}

\section{Raphael Urbani, Fabian Westermeier, Benjamin Banusch, Michael Sprung and Thomas Pfohl}

J. Synchrotron Rad. (2016). 23, 1401-1408

\section{IUCr Journals CRYSTALLOGRAPHY JOURNALS ONLINE \\ Copyright (C) International Union of Crystallography \\ Author(s) of this paper may load this reprint on their own web site or institutional repository provided that this cover page is retained. Republication of this article or its storage in electronic databases other than as specified above is not permitted without prior permission in writing from the IUCr. \\ For further information see http://journals.iucr.org/services/authorrights.html}


JOURNAL OF SYNCHROTRON RADIATION

ISSN 1600-5775

Received 7 March 2016

Accepted 4 August 2016

Edited by G. Grübel, HASYLAB at DESY, Germany

Keywords: microfluidics; X-ray scattering; photon correlation spectroscopy; flow profiles; flow geometries; particle dynamics.

Supporting information: this article has supporting information at journals.iucr.org/s

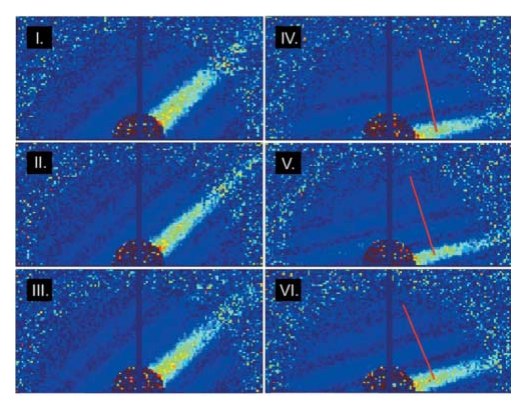

C 2016 International Union of Crystallography

\section{Brownian and advective dynamics in microflow studied by coherent $X$-ray scattering experiments}

\author{
Raphael Urbani, ${ }^{a}$ Fabian Westermeier, ${ }^{\mathrm{b}}$ Benjamin Banusch, ${ }^{\mathrm{a}}$ Michael Sprung ${ }^{\mathrm{c}}$ and \\ Thomas Pfohl ${ }^{\text {a* }}$
}

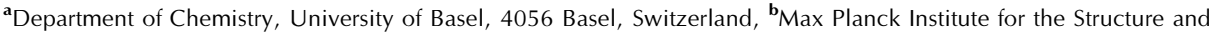
Dynamics of Matter, CFEL, 22761 Hamburg, Germany, and 'Photon Science, Deutsches Elektronen-Synchrotron, 22607 Hamburg, Germany. *Correspondence e-mail: thomas.pfohl69@gmail.com

Combining microfluidics with coherent X-ray illumination offers the possibility to not only measure the structure but also the dynamics of flowing samples in a single-scattering experiment. Here, the power of this combination is demonstrated by studying the advective and Brownian dynamics of colloidal suspensions in microflow of different geometries. Using an experimental setup with a fast two-dimensional detector and performing X-ray correlation spectroscopy by calculating two-dimensional maps of the intensity autocorrelation functions, it was possible to evaluate the sample structure and furthermore to characterize the detailed flow behavior, including flow geometry, main flow directions, advective flow velocities and diffusive dynamics. By scanning a microfocused X-ray beam over a microfluidic device, the anisotropic auto-correlation functions of driven colloidal suspensions in straight, curved and constricted microchannels were mapped with the spatial resolution of the X-ray beam. This method has not only a huge potential for studying flow patterns in complex fluids but also to generally characterize anisotropic dynamics in materials.

\section{Introduction}

In recent years, microfluidics has been a fast growing field with numerous applications in physics, biology or chemistry with a huge impact both for academic research as well as for commercial applications. Microfluidics offers a high control over the fluid characteristics and requires only small sample volumes, which makes it technologically ideally suited for microbiological and biomedical tasks, where sample quantities are often limited or expensive (Whitesides, 2006; Yager et al., 2006). Many of the experimental techniques used in microfluidics are based on optical light, such as laser Doppler velocimetry (Edwards, 1970) or particle imaging velocimetry (Adrian, 1991). During recent years, the experimental repertoire has been extended to X-ray methods, which are emerging and powerful tools for studying soft and biological materials and offer a number of advantages: the possibility to focus $\mathrm{X}$-rays to well below a micrometer offers the opportunity to probe microfluidic devices with high spatial resolution, while $\mathrm{X}$-rays allow sample structures to be probed from the atomic up to the hundreds of nanometers range. These tools enable experimental strategies, such as analyzing the evolution of reactions, self-assembly processes in concentration gradients or the response of soft systems on flow and shear (Dootz et al., 2007; Martel et al., 2008; Köster \& Pfohl, 2012; Toma et al., 2013; Trebbin et al., 2013; Weinhausen et al., 2014). The analysis of dynamical changes using X-rays is either done by 
examining changes of the scattering pattern in the course of the experiments (Merlin et al., 2011) or by spatially scanning the microfluidic device and thus transforming spatial into temporal changes (Graceffa et al., 2013).

High-brilliance third-generation synchrotrons allow for the use of coherent scattering techniques such as X-ray photon correlation spectroscopy (XPCS) (Sutton et al., 1991; Grübel \& Zontone, 2004; Bilderback et al., 2005) and thus to extract sample dynamics from the scattering patterns directly. If coherent X-rays scatter from a disordered sample, the recorded scattering intensity is additionally modulated due to interference, and shows a so-called speckle pattern. In XPCS experiments, temporal intensity fluctuations of a speckle pattern, related to changes in the spatial arrangement of the scattering objects, are evaluated. X-rays can probe a large range of momentum transfers $q$ and have a large penetration depth, which allows for probing a wide range of length scales and to analyze samples which are optically opaque (Leheny, 2012; Shpyrko, 2014). XPCS has been used for studying the dynamics of colloidal (Dierker et al., 1995; Burghardt et al., 2012; Westermeier et al., 2016), polymeric (Falus et al., 2005) and soft hybrid systems (Carnis et al., 2014; Hernández et al., 2015). Pioneering experiments on XPCS in flowing liquid samples have been performed by Fluerasu et al. (Fluerasu et al., 2008, 2010; Busch et al., 2008), studying the laminar flow in tube-like flow cells.

Here we present an XPCS study on the flow of a colloidal suspension in a more complex sample environment, namely microchannels of varying geometry. Using a two-dimensional detector for probing an expanded $q$-range, we are able to determine the colloidal particle size and shape, the anisotropic flow patterns as well as the diffusive dynamics of the system at once.

\section{Experimental}

\subsection{X-ray scattering setup}

The experiments were carried out at the coherence beamline P10 at PETRA III, Deutsches Elektronen-Synchrotron (DESY) in Hamburg, Germany. The X-rays were monochromatized to a photon energy of $8050 \mathrm{eV}$, corresponding to a wavelength of $\lambda=1.54 \AA$. The X-rays were focused using compound refractive lenses to about $5 \mu \mathrm{m} \times 5 \mu \mathrm{m}$ at the sample position. A circular beamstop with a diameter of $1.5 \mathrm{~mm}$ in front of the detector was used to block the direct beam. Measurements were recorded using a $2 \times 2$ MAXIPIX detector (Ponchut et al., 2011) with a total of $516 \times 516$ pixels of $55 \mu \mathrm{m} \times 55 \mu \mathrm{m}$ size. The detector was placed $4970 \mathrm{~mm}$ downstream of the sample, using an evacuated flight tube between sample and detector (see Fig. 1 for a sketch of the experimental setup).

\subsection{Microfluidic device and flow simulations}

The microfluidic devices were produced in analogy to the fabrication process previously described by Brennich et al. (2011). The masters for the device fabrication were produced

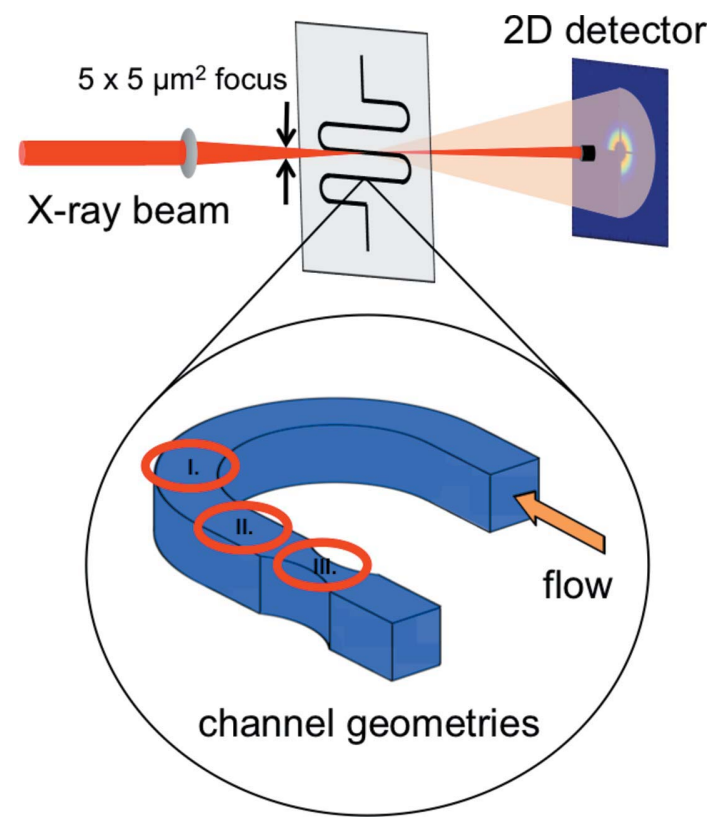

Figure 1

Experimental setup highlighting the three analyzed different microchannel geometries: curved channel (I), straight channel (II) and constriction (III)

with SU-8 negative resist (SU-8 2100, MicroChem, Newton, MA, USA). In a next step, flexible stamps were prepared by pouring PDMS and crosslinker (ratio 9:1; Sylgard 184, Dow Corning GmbH, Wiesbaden, Germany) onto the device masters and baking for $4 \mathrm{~h}$ at $80^{\circ} \mathrm{C}$. To produce the final microfluidic devices, a drop of Norland Optical Adhesive 81 (NOA 81, Norland, Cranberry, NJ, USA) was deposited on a PDMS stamp, then a piece of polystyrene foil (thickness $50 \mu \mathrm{m}$; Goodfellow Cambridge Limited, Huntingdon, UK) was placed on top of the NOA 81 drop and pressed down on the PDMS stamp. After curing under an UV lamp (366 nm, $8 \mathrm{~W}$; Camag, Muttenz, Switzerland) for about $30 \mathrm{~s}$, the device was closed by placing a second polystyrene foil, with punched holes for the tubing connections, on the open device side, gently pressing the foil onto the device and UV curing for $10 \mathrm{~min}$. The final device was mounted on a home-built sample device holder. The geometry of the microfluidic device included straight channels with a width of $150 \mu \mathrm{m}$, curved channels with a width of $150 \mu \mathrm{m}$ and a radius of curvature $R=$ $500 \mu \mathrm{m}$ and constrictions with a length of $200 \mu \mathrm{m}$ and a width of $50 \mu \mathrm{m}$; the height of the channels was $200 \mu \mathrm{m}$.

For the numerical computational fluid dynamics (CFD) simulations, we used the CFD module of the software COMSOL (Version 4.3a) to solve the incompressible NavierStokes equation in the microchannel geometries.

\subsection{Sample system}

The sample consisted of colloidal $\mathrm{SiO}_{2}$ nanoparticles with a diameter of $(160 \pm 20) \mathrm{nm}$. The particles were prepared according to a Stöber synthesis (Stöber et al., 1968) and stabilized with 3-(trimethoxysilyl)propyl methacrylate (SigmaAldrich, St Louis, MO, USA) to prevent particle agglomera- 
tion. The colloids were suspended in PPG 4000 (Sigma-Aldrich, St Louis, Missouri, USA) and driven through the device using syringe pumps (Cetoni, Korbussen, Germany). To minimize interparticle interactions, the volume fraction of the sample system was adjusted to be around 0.01 .

\subsection{XPCS and data treatment}

In coherent scattering experiments, changes in the spatial arrangement of the scattering objects, in our case the movements of nanoparticles, give rise to intensity fluctuations of the scattering pattern, which can be quantified by the intensity auto-correlation function,

$$
g_{2}(q, \tau)=\frac{\langle I(q, t) I(q, t+\tau)\rangle}{\langle I(q, t)\rangle^{2}} .
$$

Angled brackets denote a time average, $q$ is the momentum transfer, $t$ a chosen time point and $\tau$ a time shift relative to $t$. The auto-correlation functions in laminar flow depend on three independent effects: $(a)$ the intrinsic particle dynamics of the sample system, in our case the diffusive motion of the scattering objects due to Brownian motion, (b) the advective transit time of the colloids moving through the scattering volume and (c) a shear-induced oscillatory decorrelation depending on the velocity gradient of the particles (Fuller et al., 1980; Busch et al., 2008; Fluerasu et al., 2008; Burghardt et al., 2012). As the velocity difference between particles depends on the flow profile and flow direction, the correlation functions depend on the scattering geometry of the experiment and can be separated perpendicular and parallel to the (main) flow direction with

$$
g_{2, \perp}(q, \tau)=\beta \exp \left[-2 D(q) q^{2} \tau\right] \exp \left[-\left(v_{\mathrm{tr}} \tau\right)^{2}\right]+1
$$

and

$$
g_{2, \|}(q, \tau)=\beta \exp \left[-2 D(q) q^{2} \tau\right] \exp \left[-\left(v_{\mathrm{tr}} \tau\right)^{2}\right]\left[\frac{\sin \left(\Gamma_{\mathrm{s}} \tau\right)}{\Gamma_{\mathrm{s}} \tau}\right]^{2}+1
$$

Here, $\beta$ is the speckle contrast, $D(q)$ is the $q$-dependent diffusion coefficient, $v_{\text {tr }}$ the transit frequency of the particles ( $v_{\text {tr }} \simeq \bar{v} / h$; with $h$ being the beam diameter and $\bar{v}$ the mean particle velocity), and $\Gamma_{\mathrm{s}}$ is the shear relaxation rate, depending on $q$ and the mean flow velocity as $\Gamma_{\mathrm{s}}=q_{\|} \bar{v}$. Here, we have omitted the shear-dependent contribution in the flow direction to the Brownian motion as it only provides a minor correction to $g_{2, \|}(q, \tau)$. A detailed derivation of the formulas is given by Fluerasu et al. (2008) and Busch et al. (2008). Note that the functional form of the oscillatory decorrelation term for $g_{2, \|}$ in equation (3) assumes a uniform shear rate along the $\mathrm{X}$-ray beam path in the microfluidic channel. Due to the plugflow-like character along this channel dimension this approximation can be justified; however, due to the height-to-width ratio of $4 / 3$ the flow profile has still relatively strong parabolic components. While a change of the model would yield slightly different absolute flow velocities, the differences between individual measurements are constant irrespective of the chosen model, so that in the following we concentrate on relative comparisons.

Pump-driven colloids flowing through a microfluidic device with different geometric features such as constrictions and curvatures were analyzed by XPCS. Therefore, scattering pattern series of 5000 patterns at a framing rate of $300 \mathrm{~Hz}$ at different positions inside the microfluidic channels were taken. From these image series, normalized auto-correlation functions $g_{2}(\tau=3.3 \mathrm{~ms}$ to $16.66 \mathrm{~s})$ for each pixel of the detector were calculated using equation (1) and applying a $2 \times 2$ pixel binning, resulting in two-dimensional sequences (movies) of the anisotropic correlation functions for each measured position (Fig. 2a, right). While the individual scattering patterns taken at different time points $t$ are isotropic (see Fig. $2 a$, left), the two-dimensional auto-correlation patterns reveal a strong anisotropy of the dynamics. From these auto- 
correlation sequences the sample dynamics and the orientation of the flow can be obtained. Moreover, individual autocorrelation functions in the parallel and perpendicular direction at fixed $q$ values can be extracted. The subsequent perpendicular and parallel correlation functions were fitted by equations (2) and (3), respectively.

\section{Results and discussion}

To demonstrate the power of scanning coherent X-ray scattering, we analyzed the microflow of a nanoparticle suspension in microfluidic devices of different geometries. We start with the presentation of the results in straight and curved channels and proceed with the presentation of the results in a constriction.

\subsection{Flow of a nanoparticle suspension in straight and curved channels}

X-ray measurements have been performed along cross sections of both straight and curved channels. The 15 observation positions in the case of the straight channels are on a line perpendicular to the flow direction and the 17 positions in the case of the curved channels are along the radius of curvature $(R=$ $500 \mu \mathrm{m})$.

To illustrate the nanoparticle flow in straight and curved channels, we compare three selected positions along these cross sections. These positions were at $30 \%, 50 \%$ and $70 \%$ relative width of the channels for both the straight and the curved geometry as sketched in Fig. 3(a). While experiments were performed at different flow velocities, we concentrate first on experiments with a mean flow velocity of $\bar{v}=10 \mu \mathrm{m} \mathrm{s}^{-1}$.

\subsection{Small-angle $X$-ray scattering}

Fig. 2(a) (left-hand side) shows the recorded small-angle X-ray scattering (SAXS) signal of four consecutively taken patterns. Even though the sample is flowing during the experiments, the recorded patterns are isotropic, apart from parasitic scattering close to the direct beam. The azimuthally averaged intensity of the mean of all recorded frames at a fixed position is displayed in Fig. 2(b). The experimental data can be nicely modeled using the particle form factor of polydisperse spherical particles with a unimodal Schulz-Zimm size distribution, displayed by the black solid line (Aragón \& Pecora, 1976). The model yields a mean particle radius of
$\bar{R}=80 \mathrm{~nm}$ and a size polydispersity of $\Delta R / \bar{R}=0.12$. As the particles are isotropic, they show no flow alignment. The absence of additional modulations by a static structure factor shows a negligible interparticle structure in the employed sample system; the particle distribution follows a random stochastic pattern due to the small concentration of particles. The finding of a virtual absence of interparticle structures holds for all investigated velocities and microfluidic geometries like curved sections and constrictions.

\subsection{Flow direction}

The normalized two-dimensional auto-correlation patterns at a delay time $\tau=20 \mathrm{~ms}$ of the three selected positions in straight and curved channels are shown in Fig. 3(b) (see supporting information video V1 for the corresponding twodimensional auto-correlation sequences). Contrary to the SAXS patterns, these auto-correlation patterns are strongly anisotropic, nicely visualizing the additional information contained in scattering patterns with coherent illumination. A pixel with a high value of the normalized auto-correlation function indicates slow decorrelation on the respective length scale and direction. As the decorrelation is fastest in the flow (a)
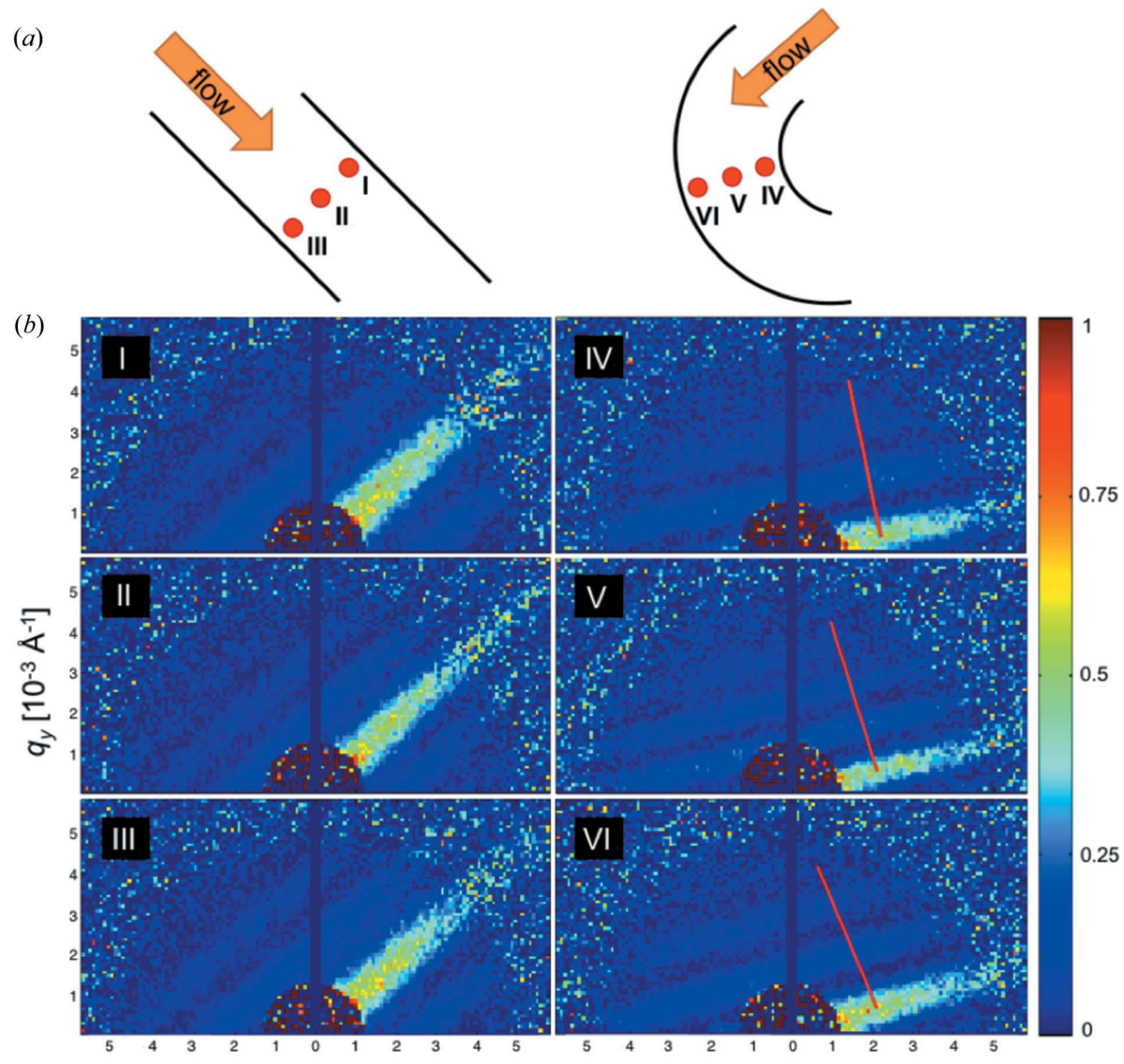

Figure 3

$$
q_{x}\left[10^{-3} \AA^{-1}\right]
$$

(a) Sketch of the selected observation positions at $30 \%, 50 \%$ and $70 \%$ relative width of the channel in straight and curved channels, respectively. (b) Correlation images of normalized $g_{2}(\tau=20 \mathrm{~ms})$ at positions I-III in a straight channel and position IV-VI in a curved channel with radius $R=500 \mu \mathrm{m}$. The average flow velocity was $10 \mu \mathrm{m} \mathrm{s}^{-1}$. The red lines in frames IV-VI are oriented parallel to the flow direction. 
direction and slowest perpendicular to it, the two-dimensional auto-correlation patterns allow for a facile extraction of the local flow orientation.

In the straight microchannel, we observe three identically oriented correlation patterns (I-III), in agreement with the parallel streamlines in straight channels at low Reynolds numbers. The overall shape of the auto-correlation pattern already shows some basic properties of the underlying dynamics. Since wider streaks correspond to lower flow velocities, the width of the streaks indicates a higher velocity in the middle of the channel (II) compared with slower velocities to the outside (I and III). The high visibility of these first- and higher-order self-beating streaks demonstrates additionally the high flow uniformity both temporally during the measurement interval and spatially in the probed scattering volume. Flow inhomogeneities would lead to a washing out of the first- and higher-order streaks.

The corresponding two-dimensional auto-correlation patterns within the curved microchannel are numbered IV to VI, where IV is the innermost position and VI the outmost one. In comparison with the straight channel section, a change of the general flow direction can be observed in the curved microchannel. This change is visualized by the red solid lines in Fig. 3(b) which are parallel to the flow direction. As a second characteristic, a change in advective velocity across the channel can be seen as well. As in the straight channel, the flow at the middle position $\mathrm{V}$ of the curved channel is the fastest, showing a narrow streak and closely packed selfbeating signals, while the advective velocities of the inner and the outer position are slower.

To quantify the flow directions at the chosen positions, the two-dimensional auto-correlation images were azimuthally integrated at a chosen lag time $\tau$. The results of these integrations are plotted as a function of the azimuthal angle in Fig. 4(a). Whereas the peak orientation for the three positions in the straight channel is identical (I-III), a change of the peak position from point IV to VI towards smaller angles, and thus a rotation of the flow orientation, is observed in the curved section.

\subsection{Sample dynamics}

The extraction of the flow direction, which is even possible when probing in a microchannel of unknown orientation as shown above, allows the auto-correlation functions parallel and perpendicular to the flow direction to be determined with high precision. Two exemplary auto-correlation functions at the same $q$ value are shown in Fig. 2(c). These auto-correlation functions were subsequently fitted using either equation (2) (perpendicular) or equation (3) (parallel to the flow direction). While the perpendicular direction is best fitted to extract the intrinsic sample dynamics, the parallel direction can be used to evaluate the flow dynamics inside the microfluidic device.

The intrinsic particle dynamics is due to Brownian motion. To obtain a quantitative value for the Brownian dynamics of the colloidal particles, auto-correlation functions perpendi-
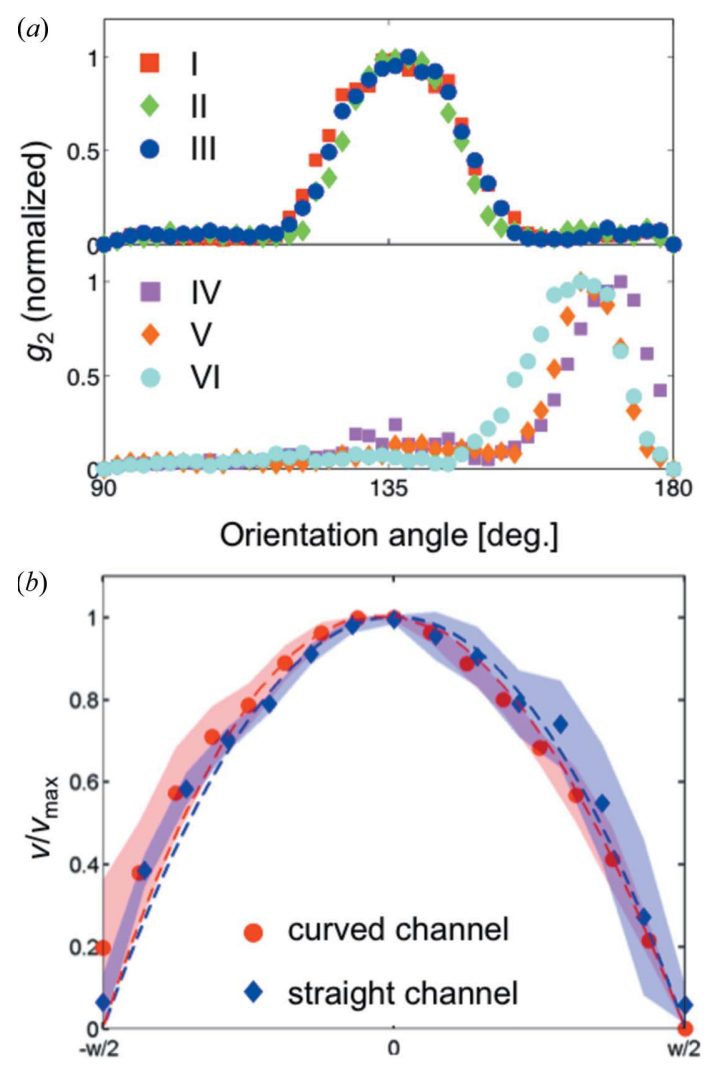

Figure 4

Relative channel position

(a) Radial integration of $g_{2}$ as a function of orientation/azimuthal angle. Numbers I-VI are related to the positions in Fig. 3(b). (b) Average flow profiles of straight (blue) and curved (red) channels. The colored area indicates the standard deviation. The velocities were normalized by $v_{\max }$ to compare experiments at different flow velocities. The dashed lines are the calculated flow profiles for the given channel dimensions.

cular to the flow direction in straight channels were fitted. As the sample shows negligible interparticle interactions (see Fig. $2 b), D(q) \simeq D_{0}$ for all analyzed $q$ values. For five different mean velocities $\bar{v}\left(2,4,6,8\right.$ and $\left.10 \mu \mathrm{m} \mathrm{s}^{-1}\right)$ an average translational free diffusion coefficient of $D_{0}=(10.5 \pm 7.1) \times$ $10^{-15} \mathrm{~m}^{2} \mathrm{~s}^{-1}$ was obtained from the experimental data. This value is in good agreement with the measured free diffusion coefficient of the same sample without any applied flow, $D_{0}=$ $(13.0 \pm 0.6) \times 10^{-15} \mathrm{~m}^{2} \mathrm{~s}^{-1}$. As the diffusion of the particles is independent of flow orientation and flow velocity, we focus in the following on the fluid dynamics due to advective flow.

$\mathrm{X}$-ray scattering series were measured at 15 to 17 positions along the line cuts depicted in Fig. 3(a) at different mean flow velocities. From each of the sequences, the auto-correlation functions parallel to the flow direction, $g_{2, \|}$, were extracted. The correlation functions were fitted using equation (3) to obtain the flow velocity at the studied position. The obtained local flow velocities along four line-scans in straight channels and three line-scans in curved channels at different mean flow velocities were normalized to the respective mean flow velocity and averaged (Fig. 4b). A parabolic flow profile was obtained for both the straight channel and the curved channel geometry, shown as colored areas in Fig. 4(b) as expected for laminar flow at low Reynolds numbers. 
The dashed lines in Fig. 4(b) show the calculated flow profiles of the corresponding channel geometries for a simple Newtonian fluid using CFD simulations. A parabolic flow profile is obtained, where the maximum flow velocity of curved channels is slightly shifted to the center of curvature. This effect can also be observed in our experimental data for the curved channels; these show a slight offset of the maximum flow velocity compared with the results of the straight channels.

\subsection{Flow of a nanoparticle suspension in microchannels with constrictions}

To further illustrate the capabilities of the combination of coherent scattering experiments and microfluidics, we investigated the effect of a constriction on the flowing nanoparticle suspension. The shape and dimensions of the studied microchannel constriction is sketched in Fig. 5(a). To exemplify the effect of the constriction, we focus first on the comparison of six different positions within the constriction: three positions of a line scan in the narrow part (I-III) and three positions in the exit of the constriction $50 \mu \mathrm{m}$ downstream of the center of the constriction (IV-VI).

Regarding the straight and curved sections, the SAXS patterns are again isotropic and reveal no further information about the anisotropy of the underlying flow dynamics. The normalized two-dimensional auto-correlation patterns at $\tau=10 \mathrm{~ms}$ of the chosen positions in the constriction are shown in Fig. 5(b) (see supplementary information video V2 for the corresponding auto-correlation sequences). The average flow velocity in the straight channel was $\bar{v}=6 \mu \mathrm{m} \mathrm{s}^{-1}$ and in the narrow constriction about three times higher (width of the entire straight channel $150 \mu \mathrm{m}$ compared with $50 \mu \mathrm{m}$ at the narrow constriction). For both line scans we selected a position in the middle of the channel (Fig. 5, II and V) and one close to each wall (I, III, IV and VI). This allowed us to directly observe the impact of the wall orientation on the flow direction.

Regarding the straight and curved channels, also for the more complex case of a constriction, the two-dimensional patterns of the auto-correlation function $g_{2}$ allow to directly obtain the orientation of the flow at the different positions. The flow direction in the narrow part (I-III) is almost identical along the three positions (i.e. across the channel). This result is in contrast to the situation further downstream (IV-VI), where we observe that the flow direction close to the walls is following the geometry of the channel. The flow at position IV is slightly tilted to the left (upwards in Fig. 5b), whereas at position VI it is tilted towards the right-hand side in the flow direction (downwards).

This observation is confirmed by the radial integration of $g_{2}$ as a function of the azimuthal angle for positions I-VI, as displayed in Fig. 6(a). Compared with positions I-III, which show a peak at almost the same orientation angle, peak maxima at different orientation angles can be observed for positions IV-VI. Moreover, the width of the streaks is a (inverse) measure of the advective flow velocity. We observe more narrow streaks (faster flow velocities) at the positions in the more narrow part (I-III) compared with the positions in the wider part (IV-VI). In addition, the flow velocities at the outer positions (I, III + IV, VI) are smaller than the corresponding center positions $(\mathrm{II}+\mathrm{V})$. This difference between the outside streams and the central streams is more pronounced
Figure 5

(a) Sketch of the microchannel constriction with six selected measurement positions. I-III are in the narrow part and IV-VI are in the wider part $50 \mu \mathrm{m}$ downstream of the center of the constriction. $(b)$ Two-dimensional correlation patterns of normalized $g_{2}(\tau=10 \mathrm{~ms})$ at positions I-III in the central most narrow part of the constriction and at positions IV-VI $50 \mu \mathrm{m}$ downstream of the center of the constriction. The average flow velocity in the straight channel before the constriction was $6 \mu \mathrm{m} \mathrm{s}^{-1}$. 
(a)

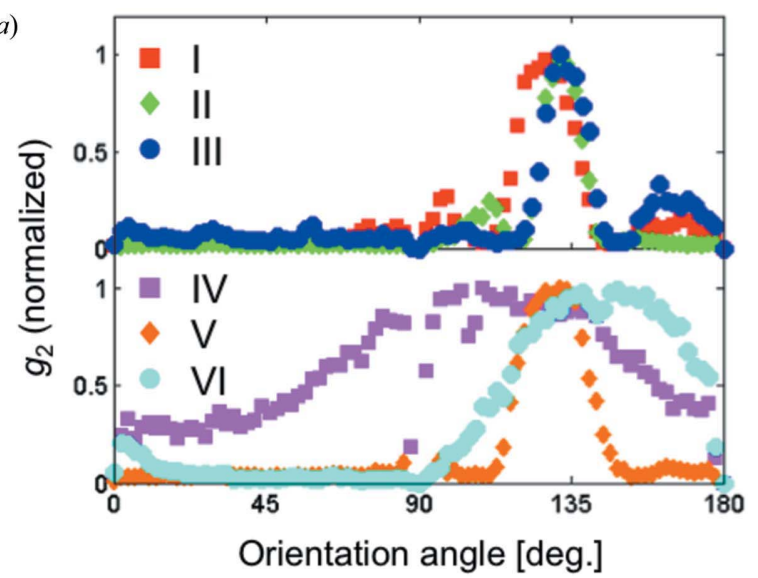

$\begin{array}{lllllllllll}\text { (b) } & 0 & 4 & 8 & 12 & 16 & 20 & 24 & 28 & 32 & \mu \mathrm{m} / \mathrm{s}\end{array}$

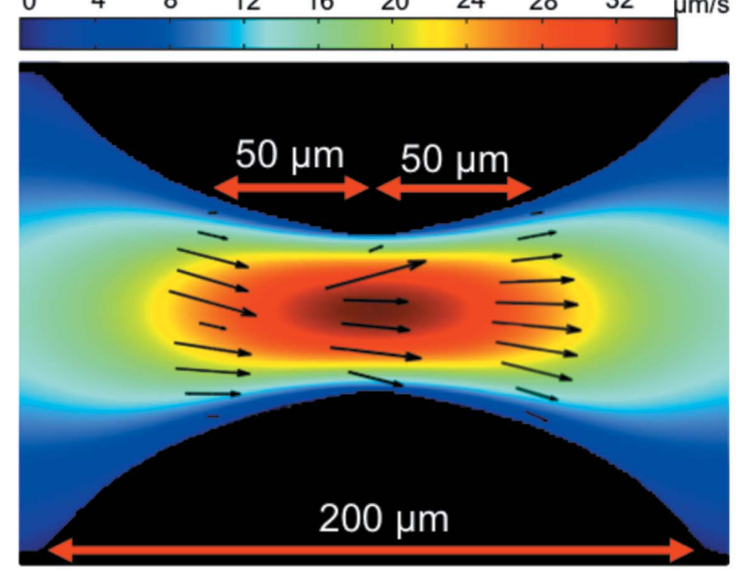

Figure 6

(a) Radial integration of $g_{2}$ as a function of orientation/azimuthal angle. Numbers I-VI are related to the positions shown in Fig. 5. (b) Calculated flow directions and flow velocities (arrow length) at three different cross sections in the constriction.

for the positions in the wider part as compared with the narrow part.

The extraction of the flow directions allowed for determining and fitting the auto-correlation functions parallel to the flow direction, which gives access to the local flow velocities. For an overview of the flow behavior in the whole constriction, local flow directions and velocities at specific positions across the most narrow part of the constriction as well as $50 \mu \mathrm{m}$ upstream and $50 \mu \mathrm{m}$ downstream are shown in Fig. 6(b). The flow directions and particle velocities along the flow were fitted from the sequences of the auto-correlation functions $g_{2}$. The flow profiles (flow speed corresponds to the lengths of the arrows) at the entrance and exit of the constriction are in good agreement with CFD simulations for a Newtonian fluid, shown as a color-coded background in Fig. 6(b). Although the fitted velocities in the center of the constriction are still in good agreement, the high flow velocities at these positions $\left(\geq 15 \mu \mathrm{m} \mathrm{s}^{-1}\right)$ make it difficult to fit the correlation functions, due to the limited framing rate of the used two-dimensional detector. The directions of the flow show congruency by pointing towards the middle of the channel at the entrance of the constriction and towards the channel walls at the exit.

\section{Conclusion and outlook}

By scanning different positions of the microfluidic devices with a microfocused X-ray beam, we are able to analyze and map the static and dynamic behavior of colloidal suspensions in straight and curved microflow as well as inside a microconstriction with high spatial resolution. While the timeaveraged scattering is isotropic and shows no differences compared with the signal of a non-flowing sample, the intensity auto-correlations of the system reveal the underlying dynamics.

Using high-framing-rate two-dimensional detectors for XPCS enables the analysis of an ample two-dimensional $q$-range by correlation image sequences, with information content beyond one of the time-averaged SAXS patterns or single correlation functions at a specific position in $q$-space. In this way, we obtain direct information about the intrinsic dynamics of the colloidal sample, which are purely due to Brownian motion and showed no changes due to the microflow within the experimental errors. In addition, the direction and the velocity of the advective flow within the scattering volume in microfluidic devices of different geometry could be detected. The auto-correlation functions at any $q_{x}, q_{y}$ values can be extracted from the correlation pattern sequences in order to quantitatively fit the dynamics of the colloidal suspension at a specific length-scale and orientation. A parabolic flow profile was found along the channel width, with a tilt towards the inner channel wall inside a curved section as expected from theory. In addition, the flow field inside a microfluidic constriction could also be accurately determined.

The rapid development of even faster two-dimensional detectors such as the LAMBDA (Pennicard et al., 2011) or the Eiger detector (Johnson et al., 2014) will allow for measuring flow dynamics and effects in more detail and with a higher temporal resolution by two-dimensional XPCS. More complex systems can be analyzed, for example the flow behavior of magnetic fluids or suspended magnetic particles, which are often opaque to optical light but of high interest in cell-biology and medicine (Plouffe et al., 2015). Other possibilities would be the flow characteristics of charged particles and associated screening effects, the dynamics of interlinked particles, or the dynamics in hierarchical self-assemblies such as the condensation of DNA (Dootz et al., 2011).

Likewise, the use of X-rays allows for the investigation of a great variety of microfluidic devices, as the need of optical transparency necessary for certain experimental techniques is obsolete. One interesting example is the lately investigated microfluidic devices based on paper, which combine low costs and are easily disposed, and might thus have a great impact on so-called point-of-care applications in medical analysis (Ren et al., 2013). Another possibility might be completely new devices such as combined microelectronic/microfluidic chips, which would naturally be based on silicon as device material.

\section{Acknowledgements}

We thank Bernd Struth for fruitful discussions and his support during initial experiments and Adriana C. Toma for her help 
on the design of the microfluidics devices. We gratefully acknowledge funding from the SNF (200020_141270). The authors declare no competing financial interest.

\section{References}

Adrian, R. J. (1991). Annu. Rev. Fluid Mech. 23, 261-304.

Aragón, S. R. (1976). J. Chem. Phys. 64, 2395-2404.

Bilderback, D. H., Elleaume, P. \& Weckert, E. (2005). J. Phys. B, 38, S773-S797.

Brennich, M. E., Nolting, J. F., Dammann, C., Nöding, B., Bauch, S., Herrmann, H., Pfohl, T. \& Köster, S. (2011). Lab Chip, 11, 708-716.

Burghardt, W. R., Sikorski, M., Sandy, A. R. \& Narayanan, S. (2012). Phys. Rev. E, 85, 021402.

Busch, S., Jensen, T. H., Chushkin, Y. \& Fluerasu, A. (2008). Eur. Phys. J. E, 26, 55-62.

Carnis, J., Cha, W., Wingert, J., Kang, J., Jiang, Z., Song, S., Sikorski, M., Robert, A., Gutt, C., Chen, S. W., Dai, Y., Ma, Y., Guo, H., Lurio, L. B., Shpyrko, O., Narayanan, S., Cui, M., Kosif, I., Emrick, T., Russell, T. P., Lee, H. C., Yu, C. J., Grübel, G., Sinha, S. K. \& Kim, H. (2014). Sci. Rep. 4, 6017.

Dierker, S., Pindak, R., Fleming, R., Robinson, I. \& Berman, L. (1995). Phys. Rev. Lett. 75, 449-452.

Dootz, R., Evans, H., Köster, S. \& Pfohl, T. (2007). Small, 3, 96-100.

Dootz, R., Toma, A. C. \& Pfohl, T. (2011). Soft Matter, 7, 8343-8351.

Edwards, R. V. (1970). J. Appl. Phys. 42, 837-850.

Falus, P., Borthwick, M. A. \& Mochrie, S. (2005). Phys. Rev. Lett. 94, 016105.

Fluerasu, A., Kwasniewski, P., Caronna, C., Destremaut, F., Salmon, J.-B. \& Madsen, A. (2010). New J. Phys. 12, 035023.

Fluerasu, A., Moussaïd, A., Falus, P., Gleyzolle, H. \& Madsen, A. (2008). J. Synchrotron Rad. 15, 378-384.

Fuller, G. G., Rallison, J. M., Schmidt, R. L. \& Leal, L. G. (1980). J. Fluid Mech. 100, 555-575.

Graceffa, R., Nobrega, R. P., Barrea, R. A., Kathuria, S. V., Chakravarthy, S., Bilsel, O. \& Irving, T. C. (2013). J. Synchrotron Rad. 20, 820-825.

Grübel, G. \& Zontone, F. (2004). J. Alloys Compd. 362, 3-11.
Hernández, R., Criado, M., Nogales, A., Sprung, M., Mijangos, C. \& Ezquerra, T. A. (2015). Macromolecules, 48, 393-399.

Johnson, I., Bergamaschi, A., Billich, H., Cartier, S., Dinapoli, R., Greiffenberg, D., Guizar-Sicairos, M., Henrich, B., Jungmann, J., Mezza, D., Mozzanica, A., Schmitt, B., Shi, X. \& Tinti, G. (2014). J. Instrum. 9, C05032.

Köster, S. \& Pfohl, T. (2012). Mod. Phys. Lett. B, 26, 1230018.

Leheny, R. L. (2012). Curr. Opin. Colloid Interface Sci. 17, 3-12.

Martel, A., Burghammer, M., Davies, R., DiCola, E., Panine, P., Salmon, J. B. \& Riekel, C. (2008). Biomicrofluidics, 2, 024104.

Merlin, A., Angly, J., Daubersies, L., Madeira, C., Schöder, S., Leng, J. \& Salmon, J.-B. (2011). Eur. Phys. J. E, 34, 1-7.

Pennicard, D., Lange, S., Smoljanin, S., Becker, J., Hirsemann, H., Epple, M. \& Graafsma, H. (2011). J. Instrum. 6, C11009.

Plouffe, B. D., Murthy, S. K. \& Lewis, L. H. (2015). Rep. Prog. Phys. 78, 016601.

Ponchut, C., Rigal, J. M., Clément, J., Papillon, E., Homs, A. \& Petitdemange, S. (2011). J. Instrum. 6, C01069.

Ren, K., Zhou, J. \& Wu, H. (2013). Acc. Chem. Res. 46, 23962406.

Shpyrko, O. G. (2014). J. Synchrotron Rad. 21, 1057-1064.

Stöber, W., Fink, A. \& Bohn, E. (1968). J. Colloid Interface Sci. 26, 62-69.

Sutton, M., Mochrie, S. G. J., Greytak, T., Nagler, S. E., Berman, L. E., Held, G. A. \& Stephenson, G. B. (1991). Nature (London), 352, 608-610.

Toma, A. C., Dootz, R. \& Pfohl, T. (2013). J. Phys. D, 46, 114001.

Trebbin, M., Steinhauser, D., Perlich, J., Buffet, A., Roth, S. V., Zimmermann, W., Thiele, J. \& Förster, S. (2013). Proc. Natl Acad. Sci. 110, 6706-6711.

Weinhausen, B., Saldanha, O., Wilke, R. N., Dammann, C., Priebe, M., Burghammer, M., Sprung, M. \& Köster, S. (2014). Phys. Rev. Lett. 112, 088102 .

Westermeier, F., Pennicard, D., Hirsemann, H., Wagner, U. H., Rau, C., Graafsma, H., Schall, P., Paul Lettinga, M. \& Struth, B. (2016). Soft Matter, 12, 171-180.

Whitesides, G. (2006). Nature (London), 442, 368-373.

Yager, P., Edwards, T., Fu, E., Helton, K., Nelson, K., Tam, M. R. \& Weigl, B. (2006). Nature (London), 442, 412-418. 